unreifen Mohnköpfe, scharf ausgetrocknet, wog 10,7 Gran, jener der reifen Mobnköpfe 27,3 Gran, so dass sich das Gewicht der Jodverbindung von den ersteren $z u$ der von den letzleren verhält wie $100 \mathrm{zu} 158$ (s. oben). Wenn nun auch die unreifen Mohnköpfe mehr Extract gaben, als die reifen, und wenn sie auch etwas mek ons aures Morphin enthielten, so muss nach Buchner's Ansicht doch die Hauptmasse der auflöslichen Bestandtheile, vom pharmokologischen Standpuncle aus betrachtet, als aus Nebenbestandtheilen bestehend erklärt werden; wogegen die reifen Mobnköpfe bei einer geringeren Ausbeute an Extract durch einen mehr bitteren Geschmack und durch eine grössere Menge des Jodniederschlags einen höheren pharmakologischen Werth bekunden. (Ann. d. Chem. $u$. Pharm. LXXIX. p. 255-259.)

G.

\title{
Ueber die Cultur des Mohns und die Gewinnung des Opiums.
}

Unter den vielen Schriften, die von Opium und dessen Gewinnung handeln, befindet sich eine von einem Deutschen, $K a ̈ m p f e r$, der uns die Art und Weise, wie man das Opium in Persien im Laufe des Sommers gewinnt, beschreibt. In die Kapseln von Papaver somniferum werden oberflächliche Einschnitte gemacht, sobald sie sich der Reife nähern, doch mit der Vorsicht, dass sie nicht ins Innere dringen; um dieses zu verhüten, bedient man sich eines Messers mit fünf Klingen, die mit einem Zuge fünf parallele Schnitle machen; der im Mohnkopfe eingeschlossene Saft fliesst dann aus und wird am nächsten Tage mit einem eigenen Instrumente abgekratzt und in ein Gefäss, welches der Arbeiter an einem Gürtel trägt, gesammelt; man macht hintereinander an allen Seiten der Kapseln solche Einschnitte. - Der so erhaltene Saft wird mit etwas Wasser angefeuchtet, umgeriuhrt und in einer hölzernen flachen Schale bis zur Consistenz und Zäbigkeit des Pechs geknetel; dann knetet man ihn noch mit den Händen und formt ihn in kleine Cylinder, die der Luft ausgesetzt werden. - In den Provinzen Kleinasiens, wie in Paphlagonien, Kappadocien, Cilicien u. a. verfahrt man nach Belon auf folgende Weise: Man säet die Samen des weissen Mohns wie bei uns die Getreidekörner und macht, wenn die Kapseln das zu der Procedur erforderliche Wachsthum erreicht haben, $d . h$. vor ihrer Reife, oberflächliche Schnitte in dieselben, aus denen einige Tropfen Saft ausfliessen, den man etwas eintrocknen lässt 
und dann abkratzt. Olivier und Charles Texier beschreiben eine fast ähnliche Methode; auf diese Weise erhält man aber das Opium stets in $T$ hränen, den Go ba ar, und nicht, wie es im Handel vorkommt. Letzteres besteht anderen Schriftstellern zufolge erstens aus einem Gemenge des Saftes, welcher aus den in die Mohnköpfe gemachten Einschnillen fliesst und eingedickt wird und aus einem Extracte, welcher nach Einigen aus einer Abkochung der Mohnköpfe, nach Anderen aus einer Abkochung verschiedener Theile der Pflanze erhalten wird, und zweitens aus einem Extracte der ganzen Pflanze.

Der Oberarzt des neapolitanischen Militairmedicinalwesens $S$ a varesi schildert uns das in Egypten gebräuchliche Verfahren, das Opium zu gewinnen. Im September oder Oclober werden die Körner gesäet, und die Pflanzen, die mit den Mlohnpflanzen hervorwuchern, entfernt; am Ende des März wird das Lnkraut yon neuem gegätet, so dass jede Mohnphanze 3 oder 4 Zoll von der andern entfernt steht.

Nach dem Blühen der Pllanzen und wenn die Kapseln saftreich geworden und ihre normale Grösse erreicht haben, werden, bevor sie sich jedoch gelb färben und hart werden, Morgens und Abends kleine Einschnitte recht dicht neben einander in sie gemacht, wozu man sich eines mit mehreren Klingen versehenen Instrumentes bedient, aber sich hüten muss, nicht in das Innere der Kapsel einzudringen.

Zehn oder zwölf Stunden, nachdem die Incisionen vollzogen worden, wird der eingedickte Saft mit einer eisernen Spatel zusammengekratzt, in ein Glas gethan, mit einigen Tropfen Wasser angefeuchtet und mit Mohnblättern bedeckt. Man wiederholt dieses Einschneiden, bis die Kapseln auf allen Seiten mit Schnitten bederkkt sind. Das ganze Verfahren kann nur 7 oder 8 Tage hindurch fortgesetzt werden. Färben sich die Köpfe gelb, so trennt man sic vom Stamme, zerstampft sie oder presst den Saft aus und zieht dann durch Ebullition den ganzen Extractivstoff aus, seihet das Decoct durch Leinwand durch und wenn es bis zu zwei Drittheilen eingedampft ist, setzt man den Saft, den man durch Ausdrücken gewonnen hat, hinzu, lässt das Ganze bis zur Extractconsistenz einkochen, fügt dann den aus den Einschnitten ausgeflossenen Saft bei und bildet kleine Brode, die man mit Pulver der grobgepulverten Blätter bestreut und an der Sonne trocknen lässt.

Wenn man aus dem aus den Schnitten fliessenden 
Safte, anstatt ihn in Schalen zu thun, eine Art von Pastillen bildet, indem man ihn auf ein ausgebreitetes, geöltes Papier fallen und an der Sonne trocknen lässt, so erhält man das Opium in Thränen, welches die Türken so sehr lieben.

Nach Landerer wird das Opium von Smyrna im Innern Kleinasiens, zumal in der Umgegend von Magnesia auf folgende Weise bereitel:

Die Pflanzen, die zum Theil von weissen, zum Theil von blauen Samen kommen, erreichen eine Höhe von $2 \frac{1}{2}$ Metres und dariber, so dass die Arbeiter, die die Einschnitle in die Kapseln machen, auf den Feldern gar nicht zu sehen sind. Die Mohnköpe sind von sehr verschiedener Grösse; damit sie so gross als möglich werden und eine rechı beträchlliche Quantität Saft enthalten, der Opium von der letzten Qualität liefert, entfernen die Pflanzer die Halfte oder zwei Drittheile dieser Köpfe; die übrigbleibenden erreichen dann eine bedeutende Grösse; diejenigen, die abgeschnitten werden, lässt man trocknen und aus den Samenkörnern wird Oel ausgepresst.

Mittelst eines gabelförmig gestalteten Instruments oder eines Messers mit gekrümmter Schneide werden parallele oder Kreuzschnitte in die Kapseln gemacht und dies so oft wiederholt, als noch Saft ausfliesst. Damit nichts von demselben verloren gehe, wenn er in grosser Menge aussickert, wird er in kleinen Austerschalen gesammelt, an der Sonne getrocknet und gesondert aufbewahrt, als die beste Sorte.

Gewöhnlich werden die Einschnitte vor Sonnenaufgang gemacht und der halbtrockne Saft Abends entfernt. Die Köpfe, die keinen Saft mehr liefern, werden abgeschnitten, in Biindel zusammengebunden und mit einem kleinen Messer geöffnet, um die Samenkörner herauszunehmen. Diese sollen, wenn sie gesäet worden, ein Opium von geringer Güte liefern, während man aus denen, die den nicht benutzlen Köpfen entnommen werden, das beste Opium gewinnt.

Die Pllanzen werden dann mit einer Sichel abgeschnitten, in Bündel gebunden und in kleine Haufen gebracht, wo sich Heerde mit kupfernen Kesseln befinden; in diesen werden sie mit Wasser gekocht und ohne filtrirt zu werden, in verschiedenen Pfannen bis zur dicken Extractconsistenz eingedamplt; ehe man dieses Extract in Brode formt, wird eine gewisse Menge von dem aus den Einschnitten erhaltenen Opium hinzufügt, das Ganze mit den Händen oder mil einem grossen Löffel geknetet, und dann 
Brode von verschiedener Grösse daraus bereitet, die in frische Mohnblätter eingewickelt werden.

Hieraus geht also hervor, dass das gewöhnlich in den Handel gelangende Opium nicht aus dem aus den Einschnitten der Köpfe ausfliessenden Safte, sondern ein Gemenge desselben und von Extracten ist, welche verschiedene Bestandtheile der Pflanze liefern. Wenn dem so ist, so muss es auffallend erscheinen, wenn das im Handel vorkommende Opium auf 100 Theile $15-16$ Theile Morphium enthält, eine Quantitäı, die der fast gleichkommt, die man in dem Safte angetroffen hat, der bloss aus den Einschnitten geflossen war.

In Deutschland haben Merk in Darmstadt und in Frankreich Brich eteau das Opium aus den Mohnköpfen dargestellt und dabei sich folgenden Verfahrens bedient: Zwei oder mehrere Frauen sind dabei beschätigl; die eine macht in die noch grünen Köpfe mittelst eines Messers mit vier Klingen Längseinschnitte, die nur die oberen Schichten der Kapsel trennen, aus diesen fliesst ein weisser Saft aus; die zweite Frau, die der ersteren in einiger Entfernung folgt, wischt mit ihrem Daumen den milchigen Saft ab und streicht ihn auf den Rand eines in der Tasche der Schürze befindlichen Glases ab. Derselbe fliesst an der Wand herunter und sammelt sich auf dem Boden des Glases an.

Zwei solcher Arbeiterinnen können in 45 Minuten $30 \mathrm{Grm}$. (1 Unze) Saft, also in einer Stunde $40 \mathrm{Grm}$ oder $400 \mathrm{Grm}$. in zehn Stunden sammeln. Diese enthaiten der Erfahrung gemäss $136 \mathrm{Grm}$. Opium.

Die Ernte ist eine reichlichere, wenn vor Sonnenaufgang oder nach Sonnenuntergang oder an nebeligen, feuchten Tagen gearbeitet wird.

Merk hat die Beobachtung gemacht, dass die Mohnköpfe in gerader Linie gepflanzt werden und weit von einander abstehen müssen, damit die Personen, die den Saft sammeln, sich gehörig bewegen können, ohne die Pflanzen zu brechen.

Grandval, oberster Pharmaceut am Hôtel-Dieu zu Rheims, bedient sich einer eigenen Methode, um aus den einheimischen Mohnköpfen Opium zu gewinnen und das auslandische, durch dessen Verfalschung dem Kranken oft nachtheilige Folgen erwachsen, entbehrlich zu machen. Er behandelt die Mohnköpfe mit Alkohol von $72 \mathrm{Grad}$, bevor sie vollständig reif sind, so dass er eine alkoholische Flüssigkeit erhält, die beim Abdampfen ein alkoholisches Extract liefert, welches, in Wasser wieder auf- 
Untersuchung der Samen etc. von Digital. purpur. 329

gelöst, zu einem wässerigen wird. Dasselbe therapeutisch angewandt, hat in der Dosis von $2 \frac{1}{2}$ Gran dieselbe Wirkung hervorgebracht, wie das exotische zu einem balben Gran. Dr. Belff.

\section{Chemische Untersuchung der Samen und Kapseln von Digitalis purpurea.}

Bekanntlich hat bereits Homolle im Jahre 1844 die Digilalis purpurea einer neuen chemischen Untersuchung unterworfen, und bei dieser Gelegenheit eine friber nicht bekannte Bereitungsmethode des Digitalin vermittelst Gerbsäure ermittelt, welche Arbeit von der Sociëté de Pharmacie mit einem Preise gekrönt wurde.

Später haben auch die Herren Kosmann, O. Hen ri, Mórin, Hervieux, Walz und Andere sich mit demselben Gegenstand beschäftigt und in neuester Zeit $\mathrm{Ho}$ molle und $Q$ ueven e die Frage zu beantworten gesucht, ob die Heilkräfte der Digitalis einzig nur von dem Digitalin oder von der Mitwirkung noch anderer Stoffe bedingt werde.

Nachdem nun die genannten Untersuchungen vorangegangen waren, hat Herr Dr. Buchner senior nun auch die Samen und Samenkapseln der Digitalis purpurea einer chemischen Untersuchung unterworfen, da über die chemische Zusammensetzung derselben bis dahin noch wenig bekannt war, und namentlich auch nicht, ob die Samen auch Digitalin enthalten, und in welcher Verbindung?

Die Hauptfragen, welche B u ch n er durch diese Untersuchung zu beantworten suchte, waren folgende:

1) Enthalten die Samen von Digilalis purpurea Digitalin in einer für therapeutische $Z$ wecke beachtenswerthen Menge?

2) Ist das fette Oel in geringer oder in beträchtlicher Menge vorhanden?

3) Gehört dieses Oel zu den schmierigen oder austrocknenden Oelen?

4) Wie verhalten sich die Samenkapseln hinsichllich ihres Digitalingehalts zu den Samen?

\section{Untersuchung der Samen.}

Zur Beantwortung dieser Fragen wurde der zerstos. sene Samen wiederholt mit Aether ausgezogen. Es wurden dadurch 40 Proc. eines fetten, austrocknenden Oels erhalten, von unangenehmem, scharfem Geruch und anfangs 